\title{
Supramolecular heterostructures formed by sequential epitaxial deposition of two-dimensional hydrogen-bonded arrays
}

\author{
Vladimir V. Korolkov ${ }^{1}$, Matteo Baldoni², Kenji Watanabe ${ }^{3}$, Takashi Taniguchi $^{3}$, Elena Besley² ${ }^{2 *}$ \\ and Peter H. Beton ${ }^{1 *}$
}

${ }^{1}$ School of Physics \& Astronomy, University of Nottingham, Nottingham NG7 2RD, UK ${ }^{2}$ School of Chemistry, University of Nottingham, Nottingham NG7 2RD, UK

${ }^{3}$ National Institute for Materials Science, 1-1 Namiki, Tsukuba, Ibaraki 305-0044, Japan

*elena.besley@nottingham.ac.uk; peter.beton@nottingham.ac.uk

Two-dimensional supramolecular arrays provide a route to the spatial control of the chemical functionality of a surface, but their deposition is in almost all cases limited to a monolayer termination. Here we investigate the sequential deposition of one 2D array on another to form a supramolecular heterostructure and realise growth, normal to the underlying substrate, of distinct ordered layers, each of which is stabilised by in-plane hydrogen bonding. For heterostructures formed by depositing terephthalic acid (TPA) or trimesic acid (TMA) on cyanuric acid/melamine (CA.M) we determine, using atomic force microscopy under ambient conditions, a clear epitaxial arrangement despite the intrinsically distinct symmetries and/or lattice constants of each layer. Structures calculated using classical molecular dynamics are in excellent agreement with the orientation, registry and dimensions of the epitaxial layers. Calculations confirm that van der Waals interactions provide the dominant contribution to the adsorption energy and registry of the layers. 
The formation of two-dimensional supramolecular arrays provides a highly flexible route to the control of the spatial organization, down to the molecular scale, of the chemical functionality of a surface ${ }^{1-4}$. These molecular networks, which can be formed through selfassembly processes on a variety of different substrates including semiconductors ${ }^{5,6}$, metals $^{7,8}$, insulators ${ }^{9-11}$ and layered materials ${ }^{12-15}$, are, in almost all cases, limited to monolayer thickness. Progress towards the growth of higher layers has so far been much more limited, with demonstrations of bilayer growth ${ }^{16,17}$ and site-specific molecular adsorption ${ }^{5,18-24}$. Specifically, the additional functional control, which may be achieved through the formation of heterostructures realized by placing one supramolecular layer on another and resulting in growth into the third dimension perpendicular to the substrate, has not been widely explored for these materials. Such additional control of material properties is well established for semiconductors, both organic ${ }^{25-30}$ and inorganic ${ }^{31}$, and, more recently, layered materials ${ }^{32}$, providing a strong motivation to explore analogue materials derived from stacked supramolecular networks. Here we describe the successful formation of heterostructures formed by the sequential growth of distinct one- and two-dimensional arrays. It is possible, using high resolution atomic force microscopy (AFM) to determine an epitaxial alignment between successive layers. Furthermore, we demonstrate using classical molecular dynamics (MD) simulations that the placement and dimensions of the layers may be predicted using well established force-fields.

We have chosen to investigate a combination of a bi-component hexagonal network (CA.M) formed by cyanuric acid (CA) and melamine (M), and mono-component honeycomb and linear arrays formed by, respectively, trimesic acid (TMA) and terephthalic acid (TPA). The heterostructures are formed by first depositing a CA.M monolayer which is then used as the 
substrate for a further deposition cycle whereby monolayers of TMA or TPA adsorbed to form a heterostructure. The layers are deposited via sequential immersion in solutions, and we have investigated heterostructure formation on the surface of hexagonal boron nitride (hBN) flakes which are exfoliated from mm-scale crystals; we have recently demonstrated that these substrates support molecular self-assembly. ${ }^{13,14}$ Since both $\mathrm{hBN}$ and, potentially, the supramolecular heterostructures are insulating, we use AFM to acquire images of the molecular arrangements in adsorbed networks. This work represents an advance in the application of AFM to imaging such networks through the acquisition of images, under ambient conditions, with sufficiently high resolution to identify the relative placement of molecules in different layers of the resulting heterostructures.

The hBN substrates typically have thicknesses of a few 10 s of nanometres and lateral dimensions of several $10 \mathrm{~s}$ of microns. Flakes are transferred to a $\mathrm{SiO}_{2} / \mathrm{Si}$ substrate and, after cleaning, molecular layers are deposited by immersion in either aqueous solutions of cyanuric acid and melamine, or ethanolic solutions of TMA or TPA. All images are acquired under ambient conditions using an Asylum Cypher AFM (full details of substrate and sample preparation are provided in Methods).

\section{Results and Discussion}

\section{Cyanuric acid-melamine on hBN}

AFM images of the CA.M network adsorbed on hBN reveal a honeycomb array (see Figure 1), very similar to that previously observed in scanning tunneling microscopy (STM) studies of CA.M on semiconductors ${ }^{33}$, graphite and metals $^{35,36}$. On hBN the array has a period 
aCA.M $=0.98 \pm 0.02 \mathrm{~nm}$, and is most clearly observed in high resolution AFM images acquired in tapping mode using the third harmonic resonant frequency of the cantilever (see Fig. 1a), in which six topographically bright features are distributed around the hexagonal unit cell. The images are consistent with the optimised structure of CA.M on hBN predicted by MD calculations (see Fig. 1b), in which junctions between cyanuric acid and melamine are each stabilized by three hydrogen bonds (more details below). The adsorbed CA.M layers are, within experimental error, structurally identical to layers of the supramolecular crystal melamine-cyanurate, which has a lattice constant of ${ }^{37} 0.96 \mathrm{~nm}$.

In intermediate resolution images (Fig. 1c) the molecular network coexists with a moiré pattern, which has a period of $6.5 \pm 0.4 \mathrm{~nm}$ and subtends an angle of $21 \pm 1^{\circ}$ with the lattice vectors of the CA.M honeycomb. The observation of a misaligned moiré pattern implies that the CA.M network is rotated with respect to the substrate. This is confirmed by contact mode AFM images (see Supplementary Information(SI), Supplementary Figure 1), in which the hBN lattice may be resolved showing a misalignment of $3 \pm 1^{\circ}$ between the $h B N$ and CA.M symmetry axes.

By varying the immersion time of the sample it is possible to control the surface coverage; larger area images show micron-scale triangular islands of CA.M on the surface (see Figs. 1d and 1e) with dimensions which increase with immersion time. This can be adjusted to a near monolayer coverage and it is also possible to form second layers, multilayers and even nanocrystals by increasing the immersion time, by repetition of immersion cycles or by increasing the concentration of the solution (see SI, Supplementary Figure 2). The large, micron-scale crystallites in Fig. 1 provide the substrate for further layers of material. 


\section{Cyanuric acid-melamine/trimesic acid heterostructures}

To explore heterostructure formation we have deposited TMA on the CA.M terminated hBN. As shown in Figure 2, TMA forms a honeycomb arrangement with domains of dimensions greater than $100 \mathrm{~nm}$ and a period $\mathrm{aTMA}_{\mathrm{T}}=1.7 \pm 0.1 \mathrm{~nm}$. The TMA arrays have a unique alignment, and images, in which both the CA.M and TMA are resolved (Fig. 2b), show that the TMA axes are rotated by $30^{\circ}$ relative to the CA.M. The TMA arrays have monolayer thickness (see profiles in Fig. 2c) and are structurally identical to the 'chickenwire' arrangement identified by Griessl et $a .^{38}$ (see Fig. 2e). Noting that there is a near coincidence of the TMA lattice period with $\sqrt{3}$ acA.M, we identify this arrangement as a $(\sqrt{3} x$ $\sqrt{3}) \mathrm{R} 30^{\circ}$ phase relative to the CA.M layer on which it is overlaid. The coverage of TMA can also be controlled by immersion time; see large area images included in SI (Supplementary Figure 3).

The observation that arrays of TMA have a very precise epitaxial relationship with the underlying CA.M network implies that the energetics of adsorption must be anisotropic and drive a specific registration. However, the interlayer interactions are expected to be dominated by van der Waals forces and to first order might be expected to be only weakly anisotropic. To explore the energetics of epitaxial growth classical MD simulations have been performed using the LAMMPS simulation package ${ }^{39}$. The OPLS potential| ${ }^{40,41}$, which was previously used to model self-assembly of CA molecules ${ }^{42}$ and molecular adsorption on 2D materials ${ }^{43}$, has been employed with additional parameters derived for CA and $\mathrm{M}$ using density functional theory as implemented in the CP2K program package ${ }^{44}$. Full computational details are included in the SI. 
The interaction of $\mathrm{CA}$ and $\mathrm{M}$ adsorbed on a model $\mathrm{hBN}$ surface was investigated computationally; hBN was treated as a rigid substrate, using Lennard-Jones (LJ) potential with parameters taken from Lee ${ }^{45}$ and with atoms fixed at their crystallographic positions. A layer of alternating $\mathrm{CA}$ and $\mathrm{M}$ molecules with a hexagonal motif was placed on top of hBN and equilibrated using MD. The predicted period of $0.975 \mathrm{~nm}$ is in excellent agreement with experiment (full details of static and dynamical simulations are included in SI). The energetics of ensembles at different orientations relative to the model CA.M substrate suggest that, in agreement with experiment, there is a reduction in energy for rotations of $\pm 2^{\circ}$ although the energy change is small, less than $\mathrm{kT} /$ molecule.

To determine the energetics driving the epitaxial alignment we overlay a rigid layer of TMA as optimised in vacuum on a rigid CA.M layer; note that the calculated lattice constant of gas phase TMA, $1.69 \mathrm{~nm}$, is almost exactly equal to the CA.M lattice constant multiplied by $\sqrt{3}$. By varying the relative orientation and position of the rigid TMA and CA.M layers we calculate the energy landscape corresponding to the adsorbed layer; a full TMA structure relaxation is then performed to ensure that the calculated energy minima represent locally stable configurations. The minimum energy orientation of the TMA is readily confirmed as $30^{\circ}$ relative to the CA.M (see discussion in SI for further details). The energy of the TMA layer in this orientation for different placement registry has also been calculated and the results are shown in Fig. 3. In short, we find energy minima when TMA molecules are placed directly above either CA or $\mathrm{M}$ molecules (see Fig $3 \mathrm{~b}$ and $3 c$ respectively); note that, due to the commensurability of the lattices, in this orientation all TMA molecules reside above equivalent sites in the CA.M layer. The energy plot in Fig. 3 also shows an energy maximum when the TMA sits above a 'pore' in the CA.M layer (i.e. at the centre of the hexagonal motif 
of CA.M). The maximum adsorption energy is calculated to be $0.79 \mathrm{eV} / \mathrm{molecule}$ and the energy barrier in moving TMA to the most unfavourable position is approximately 0.1 $\mathrm{eV} /$ molecule. The total interaction energy may be decomposed into van der Waals and Coulombic contributions, and we find (see Fig. 3) that the dominant contribution ( 90\%) to the adsorption energy comes from the van der Waals term. The energy maximum above a pore may be understood as a reduced interlayer van der Waals interaction when molecules in one layer are placed over voids in the lower layer, or, effectively, a drive towards interlayer close-packing.

Full MD simulations, in the which both the CA.M and TMA layers were flexible and allowed to move (see SI, Supplementary Figure 9 and 10), confirm that the minima identified in Fig. 3 are stable, and we find that the lowest energy occurs for TMA above M. Although we cannot distinguish CA and M molecules in our AFM images, we are able to determine experimentally that the pores of the TMA and CA.M are offset and are not coincident (thus ruling out the registry shown in Fig. $3 \mathrm{~d}$ ), which is consistent with our MD calculations (see $\mathrm{SI}$, Supplementary Figure 4 for additional images and details). Thus, the calculated structures are in excellent agreement with the dimensions, orientation and registry observed for epitaxial TMA.

\section{Cyanuric acid-melamine/terephthalic acid heterostructures}

We have also investigated the solution deposition of TPA by immersion in an ethanolic solution. This results in the formation of highly anisotropic islands with typical lengths and widths of $300 \mathrm{~nm}$ and $50 \mathrm{~nm}$ respectively (see Fig. 4a). Line profiles show that the islands 
have a height of approximately $0.3 \mathrm{~nm}$ (Fig. 4a inset) consistent with a flat lying aromatic molecule. In phase images (Fig. 4b) the rows, the background CA.M array (hexagonal array at the edges of Fig. 4b), and the CA.M/hBN moiré pattern (contrast bands at the edge of Fig. 4b) are all resolved. In addition, we observe bright lines running along the 1D islands, which are spaced by approximately $3.5 \mathrm{~nm}$ and correspond to an additional moiré pattern at the TPA/CA.M interface.

The structure of TPA and the hydrogen bonding junction, which results in the formation of $1 \mathrm{D}$ rows is shown in Fig. 4e. From Fig. $4 \mathrm{~b}$ it is possible to determine that the $1 \mathrm{D}$ rows within the TPA islands run parallel to one of the principal axes of CA.M. This is observed more clearly in contact mode AFM images (Figs. 4c and 4d); these show sequential images, which are acquired as the deflection (i.e. force) set-point is gradually increased. At low set-point (Fig. 4d), molecular resolution images of the TPA array show a separation along the rows of $\mathrm{a}_{\mathrm{TPA}}=1.00 \pm 0.02 \mathrm{~nm}$. At higher set-point (Fig. 4c), the increased tip-molecule forces result in local removal of the TPA layer, and the underlying CA.M network is resolved.

The overlay of images (Figs. $4 \mathrm{c}$ and $4 \mathrm{~d}$ ) confirm the alignment of TPA and CA.M. Moreover, there is a commensurate arrangement arising from the separation of neighbouring rows; we find that three TPA rows are accommodated in two rows of the CA.M lattice, corresponding to a distance of $\sqrt{3}$ а $_{\text {С.M }}=1.7 \pm 0.1 \mathrm{~nm}$, giving a row separation of a $_{\text {CA.M }} / \sqrt{3}=0.57 \pm 0.05 \mathrm{~nm}$. These images also show the relative positions of TPA molecules in neighbouring rows; as shown in Fig. 4e the displacement corresponds to one half of the period parallel to the row and the lattice vectors marked in Fig. 4 e are acA.m $(1 / \sqrt{3}, \pm 1 / 2)$. Consequently, TPA molecules in every sixth row are positioned above an equivalent site in the underlying CA.M giving rise to the observed interlayer moiré pattern (note every other row, and specifically every third 
row, of TPA is displaced parallel to the TPA axis by acA.M/2). The intermolecular and row separations are close to those observed for TPA on $\mathrm{Au}(111)^{46}$.

The ordering of TPA on CA.M, similar to TMA, is likely driven in part by a commensurability arising from the near match of the period of CA.M, acA.M, and the expected ${ }^{46}$ TPA molecular separation, aTPA. We have used molecular mechanics calculations to investigate the energetics of a single row comprising forty TPA molecules frozen in a configuration corresponding to the optimised gas phase structure with an intermolecular separation of $0.977 \mathrm{~nm}$. The adsorption energy at different registries and positions has been calculated, and our results show that the energy is reduced by about $0.09 \mathrm{eV} / \mathrm{molecule}$ when the row is aligned with the CA.M lattice vectors (see Fig. 5b and SI Supplementary Figures 6 and 7). An energy map for different positions of a rigid, aligned row of TPA (Fig. $5 \mathrm{~d}$ ) shows that a minimum adsorption energy occurs when the TPA is adsorbed above CA. The contributions to the total energy from van der Waals and electrostatic terms are also shown in Figure 5; as might be expected we see minima in the van der Waals contribution when the TPA is above either a $\mathrm{CA}$ or $\mathrm{M}$ molecule and a barrier region ( $0.1 \mathrm{eV} /$ molecule) when the molecules are over voids in the CA.M layer. This energy map indicates that the van der Waals contributions above $\mathrm{CA}$ and $\mathrm{M}$ molecules are comparable. However, the Coulombic terms strongly favour adsorption above a CA molecule; it is calculated to be $0.18 \mathrm{eV} /$ molecule lower above CA as compared with M. Similar energy plots are included in SI (Supplementary Figure 6) for rows in off-axis alignments. In addition, full relaxation for the TPA structures corresponding to the energy minima have been performed confirming that these arrangements are thermodynamically stable. The low energy regions near the minima extend to 0.2 to $0.4 \mathrm{~nm}$ 
in each direction allowing for some flexibility in the positioning of TPA molecules on CA.M layer, which is important in the multi-row systems.

The calculations of the energy of multiple parallel rows are more complex since the row separation does not match the periodicity of the CA.M substrate. A large number of calculations for an arrangement of 14 rows, each containing 40 TPA molecules, with both different displacements between TPA molecules in neighbouring rows and different interrow separation has been produced. Among those tested, arrangements with inter-row separation close to the experimental $(0.57 \mathrm{~nm})$ give the lowest energy, for which the adsorption energy is $-0.77 \mathrm{eV} /$ molecule. The inter-row interaction provides the energy of $0.11 \mathrm{eV} / \mathrm{molecule}$ thus compensating the destabilisation due to some of the rows not being in the preferred adsorption sites. The stability of this configuration and the preferential alignment with the CA.M layer is also confirmed by full MD simulations of the three-layered system (see a sample snapshot in Fig 5c and SI Supplementary Figure 8). The relative displacement of neighbouring rows was more difficult to capture, possibly due to an intrinsic limitation of the classical potential; several alternative models were explored as described in SI. Overall, there is good agreement between computational predictions and our observations of both TMA and TPA alignment, periodicity and registry.

Our work demonstrates that supramolecular heterostructures can be formed by sequential deposition of layers with different structural and optical properties. For the systems studied here there is a well-defined epitaxial relationship between the grown layers and we can understand many of the observed properties using standard molecular dynamics simulations. Although the interlayer interactions are dominated by van der Waals terms, which are not intrinsically directional, an orientational relationship emerges driven by the 
energy minimization arising from maximizing interlayer close-packing. Additional terms arising from Coulombic interactions determined by the relative positions of polar groups within the two layers are also significant in controlling the precise registry. There are therefore good prospects for predicting structural behaviour in analogue heterostructures between other materials.

In the future, we envisage that this approach to supramolecular heterostructures will provide the foundation for the growth of much more complex materials, in which multiple layers can be sequentially deposited with the possibilities of tuning the chemical, optical and electronic properties of the resulting heterostructure. The exploitation of hydrogen bonding stabilizes the growth of two-dimensional sheets, which have highly parallel interfaces. The use of $\mathrm{hBN}$ as a substrate suggests that this approach can be combined with 2D materials to introduce molecular functionality into stacked device architectures ${ }^{32}$, and may also provide a complete molecular analogue approach to the stacking of layered materials.

\section{Methods}

hBN flakes were mechanically exfoliated onto $\mathrm{Si} / \mathrm{SiO}_{2}$ substrates and briefly flame annealed prior to adsorption experiments. Cyanuric acid-melamine monolayers on hBN were prepared via adsorption from $8 \mu \mathrm{M}$ aqueous solution of melamine cyanurate. The melamine cyanurate solution was prepared from equal amounts of $8 \mu \mathrm{M}$ solutions of melamine $(99 \%$, Sigma-Aldrich, Gillingham, Dorset, UK) and cyanuric acid ( $\geq 98 \%$, Sigma-Aldrich, Gillingham, Dorset, UK). 
TMA was deposited on CA.M from $20 \mu \mathrm{M}$ and TPA from $60 \mu \mathrm{M}$ ethanolic solutions. The adsorption time for TMA on CA.M was 5 minutes and for TPA 20 minutes. The deposition was performed directly after the CA.M complex was deposited on hBN flakes. After the deposition the surface was blown dry with nitrogen for $\sim 1 \mathrm{~min}$. The samples were then immediately transferred to AFM for imaging.

AFM images were acquired with a Cypher AFM (Oxford Instruments - Asylum Research, Santa Barbara, USA) in both AC and contact modes using Multi75AI-G (Budget Sensor, Bulgaria) cantilevers with a nominal spring constant of $\sim 2.8 \mathrm{~N} / \mathrm{m}$ and a fundamental resonant frequency of $\sim 75 \mathrm{kHz}$ and higher harmonics $\sim 415 \mathrm{kHz}\left(2^{\text {nd }}\right.$ harmonic $)$ and $\sim 1280 \mathrm{kHz}$ ( $3^{\text {rd }}$ harmonic). We have also used NuNano Scout 350 probes with a nominal spring constant of $42 \mathrm{~N} / \mathrm{m}$ and resonant frequency of $\sim 350 \mathrm{kHz}$ (Nu Nano Ltd, Bristol, United Kingdom) and high-frequency Arrow UHF probes (NanoWorld AG, Neuchâtel, Switzerland) with a mean resonant frequency of $\sim 2 \mathrm{MHz}$. All imaging was performed in the ambient without any temperature or environmental control.

AFM images were analysed and extracted with WSXM software ${ }^{47}$ (WSxM solutions, www.wsxmsolutions.com).

Following publication the AFM images from experiments on which this paper is based will be made publicly accessible $e^{48}$.

\section{Author Contributions}


V.V.K. and P.H.B conceived and designed the experiments which were carried out by V.V.K.; the hBN crystals were grown by K.W. and T.T.; the numerical calculations were conceived through a discussion between M.B., E.B., P.H.B. and V.V.K and were carried out by M.B. with additional input from E.B.; the paper was written by P.H.B., V.V.K , M.B. and E.B with revisions and comments from all authors.

\section{Acknowledgements}

We are very grateful to the Leverhulme Trust for providing financial support under grant RPG-2016-104 and to the Engineering and Physics Sciences for support through grant EP/N033906/1. EB gratefully acknowledges receipt of an ERC Consolidator Grant.; MB and EB acknowledge the University of Nottingham and Midplus High Performance Computing facilities for providing computational time. K.W. and T.T. acknowledge support from the Elemental Strategy Initiative conducted by the MEXT, Japan and JSPS KAKENHI Grant Numbers JP26248061, JP15K21722 and JP25106006. 


\section{References}

1. Elemans, J. A. A. W., Lei, S. \& De Feyter, S. Molecular and supramolecular networks on surfaces: From two-dimensional crystal engineering to reactivity. Angew. Chemie Int. Ed. 48, 7298-7333 (2009).

2. Macleod, J. M. \& Rosei, F. Molecular self-assembly on graphene. Small 10, 1038-1049 (2014).

3. Slater (née Phillips), A. G., Beton, P. H. \& Champness, N. R. Two-dimensional supramolecular chemistry on surfaces. Chem. Sci. 2, 1440 (2011).

4. Wang, D., Wan, L.-J. \& Bai, C.-L. Formation and structural transition of molecular selfassembly on solid surface investigated by scanning tunneling microscopy. Mater. Sci. Eng. R-Rep 70, 169-187 (2010).

5. Theobald, J. A., Oxtoby, N. S., Phillips, M. A., Champness, N. R. \& Beton, P. H. Controlling molecular deposition and layer structure with supramolecular surface assemblies. Nature 424, 1029-31 (2003).

6. Baris, B. et al. Robust and open tailored supramolecular networks controlled by the template effect of a silicon surface. Angew. Chemie Int. Ed. 50, 4094-4098 (2011).

7. Barth, J. V et al. Building Supramolecular Nanostructures at Surfaces by Hydrogen Bonding. Angew. Chemie Int. Ed. 1230-1234 (2000).

8. Barth, J. V, Costantini, G. \& Kern, K. Engineering atomic and molecular nanostructures at surfaces. Nature 437, 671-9 (2005).

9. Maier, S. et al. Nanoscale engineering of molecular porphyrin wires on insulating surfaces. Small 4, 1115-8 (2008).

10. Burke, S., Mativetsky, J., Hoffmann, R. \& Grütter, P. Nucleation and Submonolayer Growth of C60 on KBr. Phys. Rev. Lett. 94, 96102 (2005). 
11. Rahe, P. et al. Tuning molecular self-assembly on bulk insulator surfaces by anchoring of the organic building blocks. Adv. Mater. 25, 3948-56 (2013).

12. Li, B. et al. Self-assembled air-stable supramolecular porous networks on graphene. ACS Nano 7, 10764-72 (2013).

13. Korolkov, V. V et al. Bimolecular porous supramolecular networks deposited from solution on layered materials: graphite, boron nitride and molybdenum disulphide. Chem. Commun. 50, 8882-5 (2014).

14. Korolkov, V. V et al. van der Waals-Induced Chromatic Shifts in Hydrogen-Bonded Two-Dimensional Porphyrin Arrays on Boron Nitride. ACS Nano 9, 10347-10355 (2015).

15. Ludwig, C., Gompf, B., Petersen, J., Strohmaier, R. \& Eisenmenger, W. STM investigations of PTCDA and PTCDI on graphite and MoS2. A systematic study of epitaxy and STM image contrast. Zeitschrift fur Phys. B Condens. Matter 93, 365-373 (1994).

16. Blunt, M. O. et al. Guest-induced growth of a surface-based supramolecular bilayer. Nat. Chem. 3, 74-8 (2011).

17. Ciesielski, A. et al. Self-templating 2D supramolecular networks: a new avenue to reach control over a bilayer formation. Nanoscale 3, 4125 (2011).

18. Xu, B., Tao, C., Cullen, W. G., Reutt-Robey, J. E. \& Williams, E. D. Chiral symmetry breaking in two-dimensional C60-ACA intermixed systems. Nano Lett. 5, 2207-2211 (2005).

19. Wei, Y. \& Reutt-Robey, J. E. Directed Organization of C 70 Kagome Lattice by Titanyl Phthalocyanine Monolayer Template. J. Am. Chem. Soc. 133, 15232-15235 (2011).

20. Skomski, D. \& Tait, S. L. Interfacial organic layers for chemical stability and crystalline 
ordering of thiophene and carboxyl films on a metal surface. J. Phys. Chem. C 118, 1594-1601 (2014).

21. Chen, W. et al. Orientationally Ordered C 60 on p-Sexiphenyl Nanostripes on. 2, 693698 (2008).

22. Huang, H. et al. Molecular orientation of CuPc thin films on $\mathrm{C}[\mathrm{sub} 60] / \mathrm{Ag}(111)$. Appl. Phys. Lett. 94, 163303-163304 (2009).

23. Yoshimoto, S., Sawaguchi, T., Su, W., Jiang, J. \& Kobayashi, N. Superstructure formation and rearrangement in the adlayer of a rare-earth-metal triple-decker sandwich complex at the electrochemical interface. Angew. Chemie Int. Ed. 46, 10711074 (2007).

24. Yoshimoto, S. et al. Controlled molecular orientation in an adlayer of a supramolecular assembly consisting of an open-cage C60 derivative and ZnII octaethylporphyrin on Au(111). Angew. Chemie Int. Ed. 43, 3044-3047 (2004).

25. Hinderhofer, A. \& Schreiber, F. Organic-organic heterostructures: Concepts and applications. ChemPhysChem 13, 628-643 (2012).

26. Forker, R. et al. Electronic decoupling of aromatic molecules from a metal by an atomically thin organic spacer. Adv. Mater. 20, 4450-4454 (2008).

27. Gruenewald, M. et al. Commensurism at electronically weakly interacting phthalocyanine/PTCDA heterointerfaces. Phys. Rev. B 91, 155432 (2015).

28. Chen, W., Qi, D. C., Huang, H., Gao, X. \& Wee, A. T. S. Organic-organic heterojunction interfaces: Effect of molecular orientation. Adv. Funct. Mater. 21, 410-424 (2011).

29. Schmitz-Hübsch, T. et al. Direct observation of organic-organic heteroepitaxy: perylenetetracarboxylic-dianhydride on hexa-peri-benzocoronene on highly ordered pyrolytic graphite. Surf. Sci. 445, 358-367 (2000). 
30. Schwarze, M. et al. Band structure engineering in organic semiconductors. Science (80-. ). 352, 1446-1449 (2016).

31. Orton, J. \& Foxon, T. Molecular Beam Epitaxy: A Short History. (Oxford University Press, 2015).

32. Geim, A. K. \& Grigorieva, I. V. Van der Waals heterostructures. Nature 499, 419-25 (2013).

33. Perdigão, L. M. A., Champness, N. R. \& Beton, P. H. Surface self-assembly of the cyanuric acid-melamine hydrogen bonded network. Chem. Commun. 538-540 (2006).

34. Zhang, X., Chen, T., Chen, Q., Wang, L. \& Wan, L.-J. Self-assembly and aggregation of melamine and melamine-uric/cyanuric acid investigated by STM and AFM on solid surfaces. Phys. Chem. Chem. Phys. 11, 7708-7712 (2009).

35. Staniec, P. A., Perdigão, L. M. A., Rogers, B. L., Champness, N. R. \& Beton, P. H. Honeycomb Networks and Chiral Superstructures Formed by Cyanuric Acid and Melamine on Au(111). J. Phys. Chem. C 111, 886-893 (2007).

36. Xu, W. et al. Cyanuric acid and melamine on $\mathrm{Au}(111)$ : Structure and energetics of hydrogen-bonded networks. Small 3, 854-858 (2007).

37. Ranganathan, A., Pedireddi, V. R. \& Rao, C. N. R. Hydrothermal Synthesis of Organic Channel Structures: 1:1 Hydrogen-Bonded Adducts of Melamine with Cyanuric and Trithiocyanuric Acids. J. Am. Chem. Soc. 121, 1752-1753 (1999).

38. Griessl, S., Lackinger, M., Edelwirth, M., Hietschold, M. \& Heckl, W. M. Self-Assembled Two-Dimensional Molecular Host-Guest Architectures From Trimesic Acid. Single Mol. 3, 25-31 (2002).

39. Plimpton, S. Fast parallel algorithms for short-range molecular dynamics. J. Comput. 
Phys. 117, 1-19 (1995).

40. Jorgensen, W. L. \& Tirado-Rives, J. The OPLS [optimized potentials for liquid simulations] potential functions for proteins, energy minimizations for crystals of cyclic peptides and crambin. J. Am. Chem. Soc. 110, 1657-1666 (1988).

41. Jorgensen, W. L., Maxwell, D. S. \& Tirado-Rives, J. Development and Testing of the OLPS All-Atom Force Field on Conformational Energetics and Properties of Organic Liquids. J. Am. Chem. Soc. 118, 11225-11236 (1996).

42. Kannappan, K., Werblowsky, T. L., Rim, K. T., Berne, B. J. \& Flynn, G. W. An experimental and theoretical study of the formation of nanostructures of selfassembled cyanuric acid through hydrogen bond networks on graphite. J. Phys. Chem. B 111, 6634-6642 (2007).

43. Conti, S. \& Cecchini, M. Accurate and Efficient Calculation of the Desorption Energy of Small Molecules from Graphene. J. Phys. Chem. C 119, 1867-1879 (2015).

44. Hutter, J., Iannuzzi, M., Schiffmann, F. \& Van de Vondele, J. atomistic simulations of condensed matter systems. Wiley Interdiscip. Rev. Comput. Mol. Sci. 4, 15-25 (2014).

45. Lee, J. H. A Study on a Boron-Nitride Nanotube as a Gigahertz Oscillator. J. Korean Phys. Soc. 49, 172-176 (2006).

46. Clair, S. et al. STM Study of Terephthalic Acid Self-Assembly on Au(111): HydrogenBonded Sheets on an Inhomogeneous Substrate. J. Phys. Chem. B 108, 14585-14590 (2004).

47. Horcas, I. et al. WSXM: A software for scanning probe microscopy and a tool for nanotechnology. Rev. Sci. Instrum. 78, 13705 (2007).

48. Nottingham Research Data Management. 10.17639/nott.316 

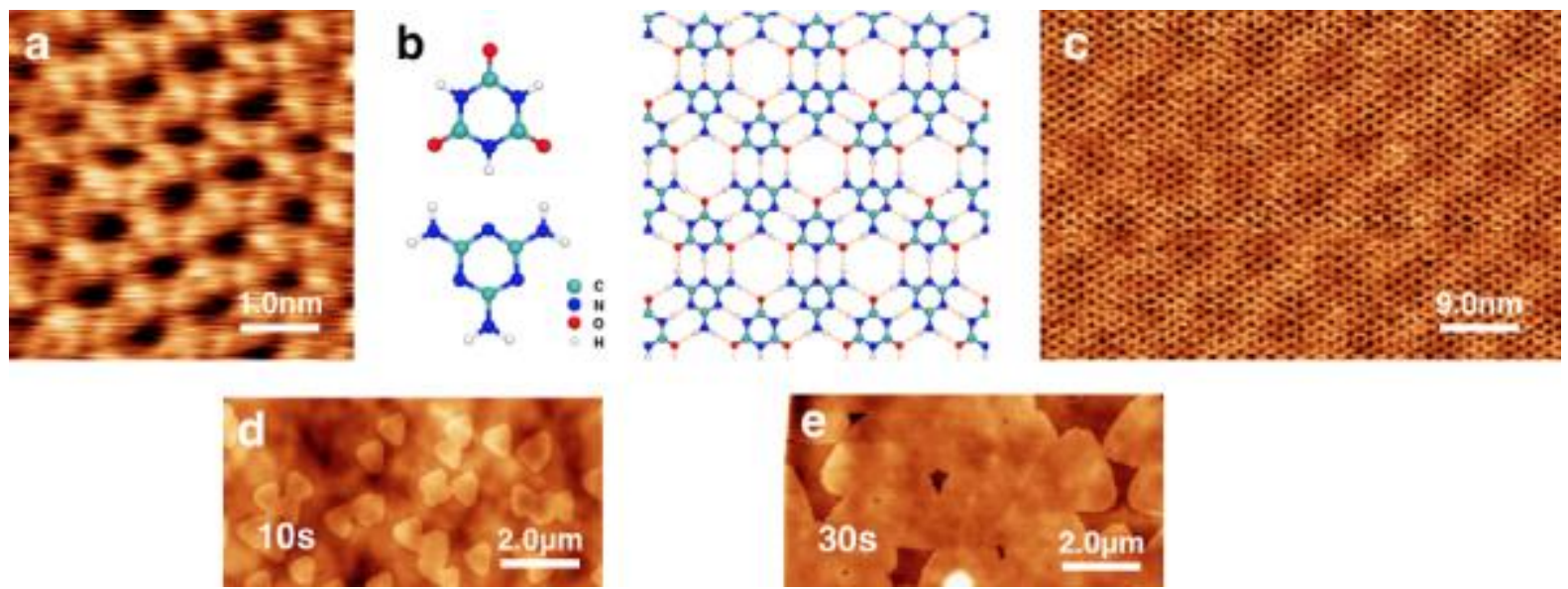

Figure 1 Adsorption of CA.M on hBN. a) tapping mode image acquired using third harmonic resonance showing the honeycomb CA.M network with a period of $0.98 \pm 0.02 \mathrm{~nm}$; b) fully optimised molecular structures of cyanuric acid (top left), melamine (lower left), and the CA.M honeycomb arrangement which is stabilised by three hydrogen bonds between neighbouring molecules; c) AFM image showing a moiré pattern with period $6.5 \pm 0.4 \mathrm{~nm}$ formed by CA.M on hBN - the angle between the principal axes of the CA.M and the moiré pattern is $21 \pm 1^{\circ}$; d), e) large area images showing variation in coverage and island size for different immersion times of 10 s (d) and 30 s (e). 


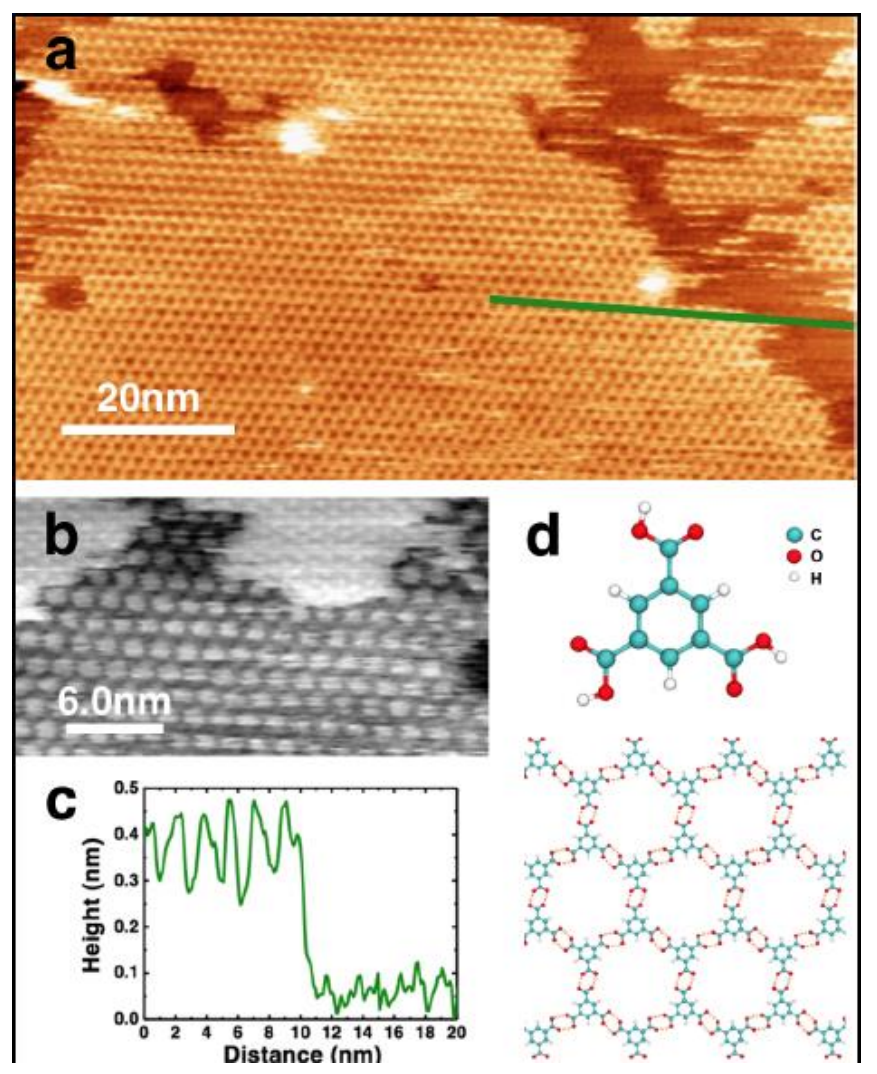

Figure 2 Heterostructures of CA.M and trimesic acid. a) large area AFM image showing a honeycomb ('chickenwire') arrangement of TMA (bright contrast) on CA.M (lower contrast). b) higher resolution image in reverse contrast chosen to highlight the alignment between TMA (lower part) and CA.M - array in the brighter regions in the upper part of the image; the principal axes of TMA are rotated relative to CA.M by $30^{\circ}$. c) profile along green line in a) showing a height of $\sim 0.35 \mathrm{~nm}$ of the TMA layer. d) structure of TMA and the honeycomb TMA network. 

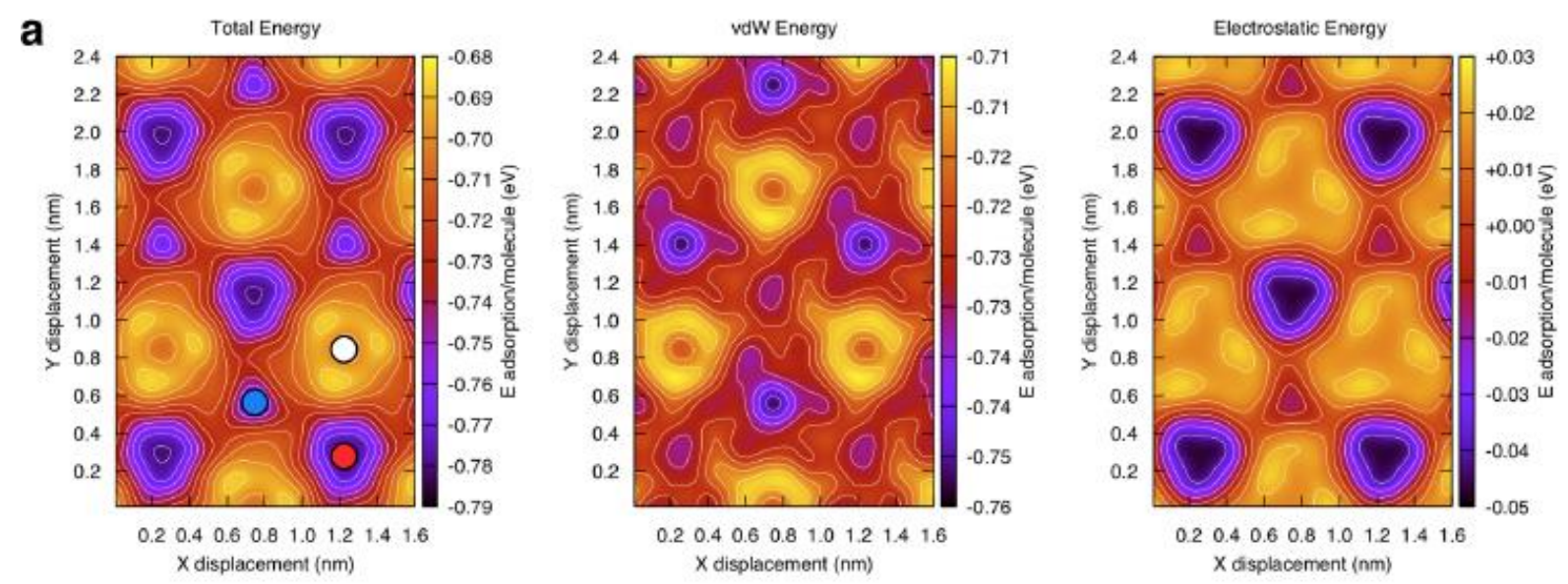

b

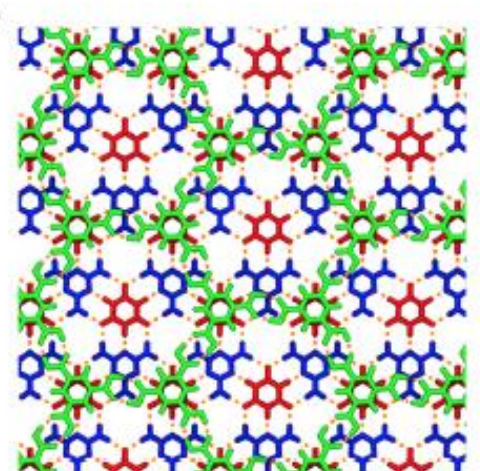

c

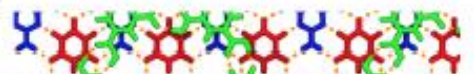

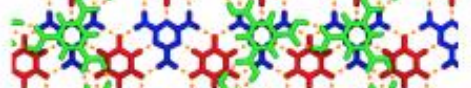

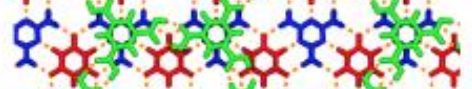

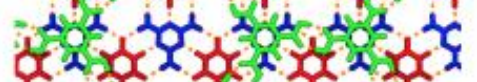

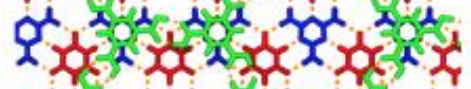

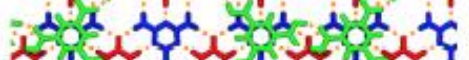

d

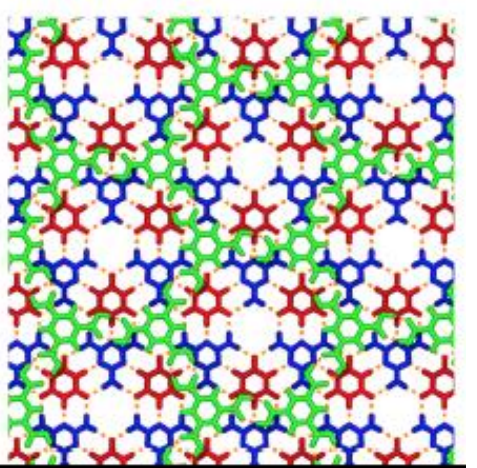

Figure 3 Energetics of the CA.M/TMA heterostructure. a) Lateral slide analysis of the total potential energy landscape for a TMA network as optimised in vacuum on CA.M in the preferential mutual orientation $\left(30^{\circ}\right)$, showing the total energy (left), as well as the van der Waals (centre) and electrostatic contributions (right) to the energy as a function of the relative placement of the TMA layer over the CA.M. b)-d) show the fully optimised TMA network corresponding to the registries highlighted by, respecteively, the red (b), blue (c) and white (d) dots in a). The configurations in b) and c) correspond to a TMA sitting directly over a CA or, respecteively, $\mathrm{M}$ molecule; these positions maximise the van der Waals contributions to the energy. In d) a TMA is directly over a void; this is an energetically unfavourable position. TMA, CA and MA are coloured in green, red and blue respectively. See SI for additional details and different orientations. 


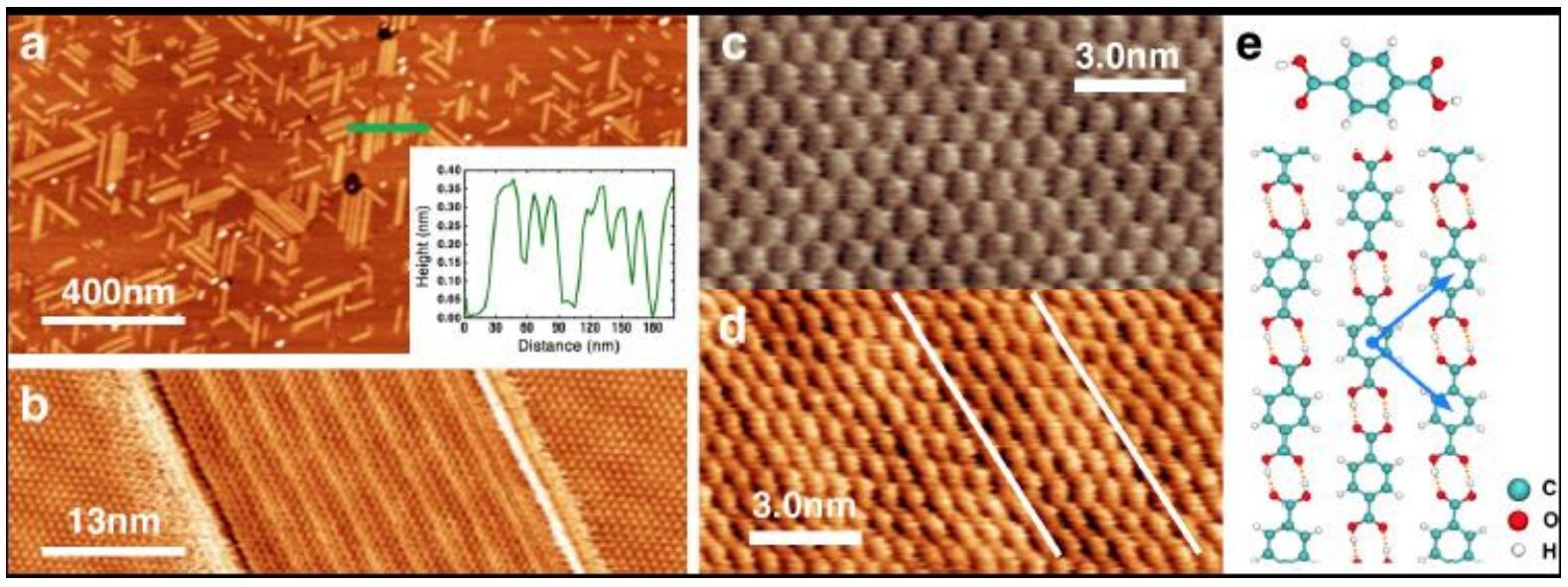

Figure 4 TPA/CA.M supramolecular heterostructure. a) AFM image showing 1D crystallites of TPA adsorbed on a near-monolayer coverage of CA.M; inset - height profile along line marked in green. The topographic height of the island is approximately $0.3 \mathrm{~nm}$ as expected for a monolayer. b) a phase image of a single TPA crystallite in which the TPA molecules, underlying CA.M network and the CA.M/hBN moiré pattern can all be resolved. The alignment of the TPA rows with the principal axis of CA.M is clearly resolved in this image. A contrast variation between TPA rows occurs due to a commensurability between CA.M and TPA; the separation of bright rows is approximately $3.4 \mathrm{~nm}$ corresponding to six rows. $\mathrm{c}$ ) and d) show contact mode images of the same area acquired at low setpoint (d) where the TPA rows are resolved, and at higher deflection setpoint (c) where the underlying CA.M is revealed through removal of the TPA; in combination c) and d) show that every sixth TPA row is positioned over every fourth CA.M row. e) molecular structure of TPA, and TPA rows stabilised by hydrogen bonding as optimised in vacuum. The lattice vectors of the TPA overlayer are represented by blue arrows and correspond to (aсA.m/v3, \pm acA.m/2). 
a

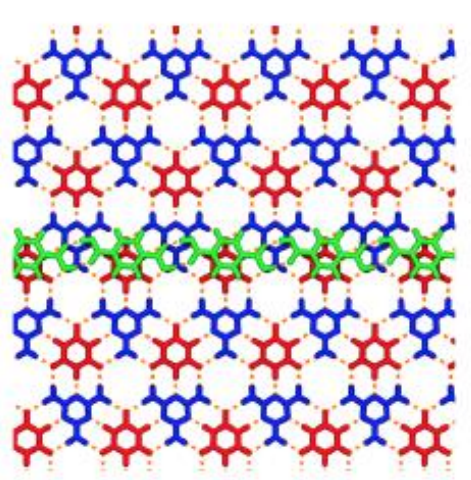

d

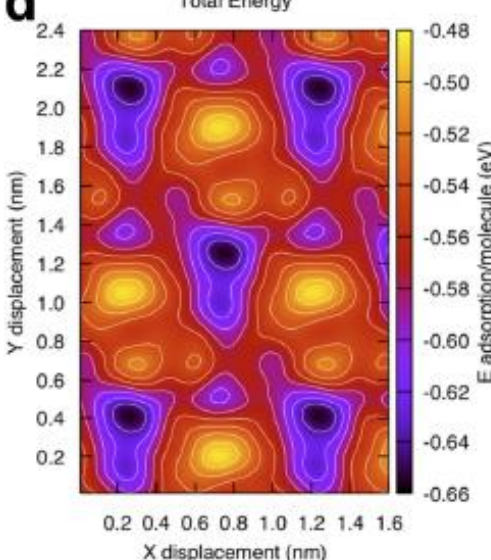

b
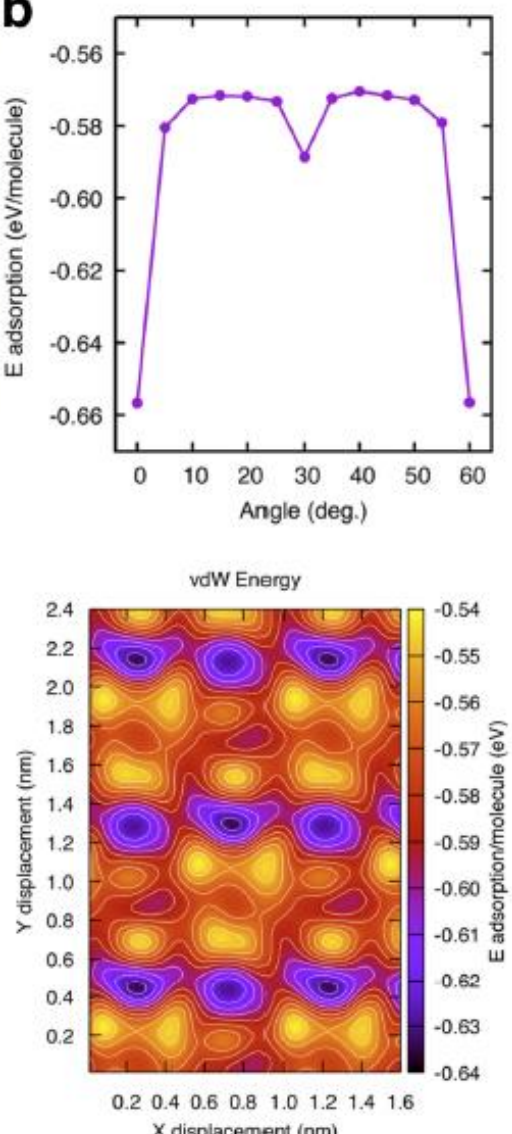
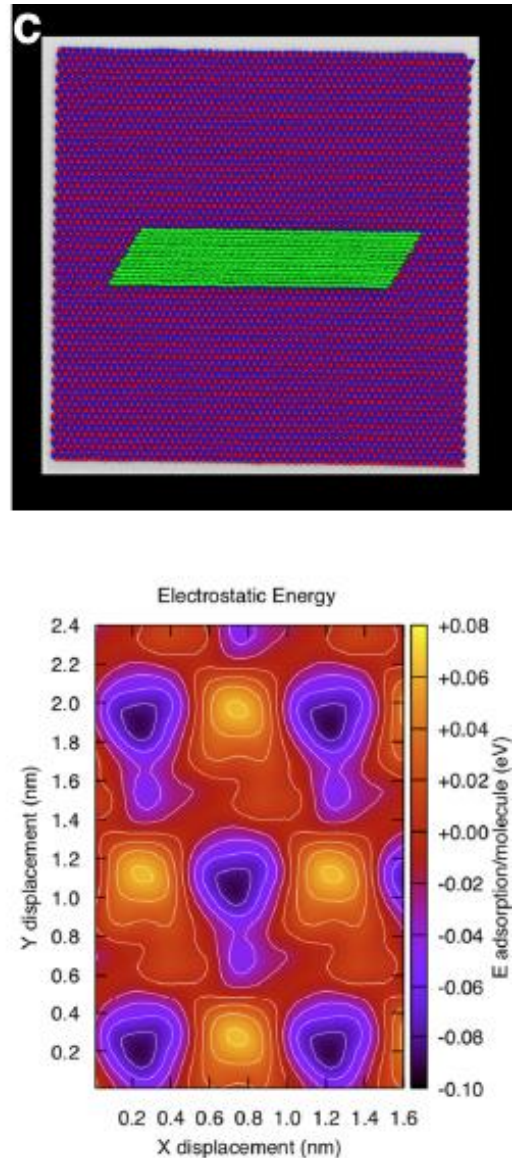

Figure 5 Energetics of the CA.M/TPA heterostructure. a) fully optimised TPA row corresponding to the global energy minimum. b) minimum of the adsorption energy as a function of the angle between the principal axis of the TPA row and the CA.M lattice vector. c) a snapshot from MD simulations of 14 TPA rows on CA.M/hBN in the preferential orientation d) lateral slide analysis of the total potential energy landscape for a TPA row as optimised in vacuum on CA.M in the preferential mutual orientation $\left(0^{\circ}\right)$ including van der Waals and electrostatic energy contributions. TMA, CA and MA are coloured in green, red and blue respectively. See SI for additional details and different orientations. 